\title{
Treadmill vs Cycling in Parkinson's disease rehabilitation: Commentary on "Intensive cycle ergometer training improves gait speed and endurance in patients with Parkinson's disease: A comparison with treadmill training" by Arcolin et al., 2016
}

\author{
Miguel Fernández-del-Olmo* \\ Department of Physical Education and Sport, Faculty of Sciences of Sport and Physical Education, \\ University of A Coruña, Pazos-Liáns, Oleiros, A Coruña, Spain
}

In a recent paper from Restorative Neurology and Neuroscience, Arcolin and colleagues (2016) report the effects of treadmill and cycle ergometer training in Parkinson's disease (PD-T and PD-C, respectively). After 3 weeks of training, both PD-T and PD-C groups improved walking parameters and reduced the clinical signs of PD. The authors suggest that the cycle ergometer is a valid alternative to treadmill in patients with PD, particularly when performing home exercises. Although, this is an interesting study from a rehabilitation point of view, the author's interpretation of the results is questionable.

First, the authors point out that their findings, showing gait improvements as a result of cycle ergometer training, contrast with a previous study (Lauhoff et al., 2013) that did not find an improvement in walking after a cycle training program. Arcolin and colleagues argued that the higher

\footnotetext{
${ }^{*}$ Corresponding author: Miguel Fernández-del-Olmo, Department of Physical Education and Sport, Faculty of Sciences of Sport and Physical Education, University of A Coruña, Av. Ernesto Che Guevara 121, Pazos-Liáns, 15179 Oleiros, A Coruña, Spain. Tel.: +34 981167000; Fax: +34 981167048; E-mail: mafo@udc.es.
}

intensity training that was used in their study in comparison with Lauhorff et al.'s may explain these differences. However, in Arcolin et al.'s study the PD participants performed common exercises for 60 minutes in addition to the cycle or treadmill training sessions. These exercises included balance and transfer tests such as "sit-to-stand" and "getting in/out of bed". It is likely that these exercises may have also contributed to the observed gait improvements since it has been reported that balance training can improve postural instability (Smania et al., 2010) and gait in PD (Wong-Yu \& Mak, 2015; Yang et al., 2015). In addition, higher intensity training does not necessarily result in greater gait improvements in PD subjects (Shulman et al., 2013). Thus, it is difficult to elucidate whether the gait improvements observed in the study of Arcolin et al. are due to the cycling or treadmill training, the common exercises, or a combination of both. The same question can be formulated for all the remaining outcome measurements.

In addition, the authors state (page 133) that "cycle ergometer or treadmill is useful for improving walking distance and gait pattern”. Assuming a null effect 
of the common exercises discussed above, this seems to be true only for the walking distance. A detailed exploration of the changes in the gait parameters raises several doubts about the effects of the cycle ergometer on the gait pattern in PD. Gait pattern in PD patients is characterized by an inability to generate appropriate stride length, while cadence control in intact and is used as a compensatory mechanism (Morris et al., 1996). Patients from the PD-T and PD-C groups showed significant increases of both the stride length and speed but only the PD-C group showed an increase in their cadence. The authors stressed that the changes in cadence were negligible. However, any interpretation of the changes in cadence must also take in to account the changes in stride length and speed, especially when the gait pattern is being evaluated. When this procedure is followed one arrives at a different interpretation of the results. Arcolin and colleagues reported that the increments in cadence in both groups were around $4 \%$ but that the step length improvements were approximately $7 \%$ and $13 \%$ and speed improvements $12 \%$ and $19 \%$ for PD-C and PD-T, respectively. Therefore, it seems that the changes in speed in the PD-T group were mainly due to an increase in the stride length but not in the cadence. In the PD-C group the increment in speed was lower in comparison with the PD-T group, and was due to a significant increase in both stride length and cadence. In other words, although both groups increased their speed, the treadmill training lead to a more normalized gait pattern compared with cycle training. These results support previous findings that showed a specific gait enhancement associated with treadmill walking in PD subjects (Bello et al., 2013). Whether the changes in the gait pattern associated with cycle ergometer training result from specific effects in PD or from general benefits associated with this exercise remain to be explored. This question is of relevance, when determining the most appropriate therapeutic exercise program for PD subjects.

In summary, cycle ergometer training seems to induce beneficial effects in PD. The ease of its implementation in a home environment makes it a potential tool for PD rehabilitation. However, in view of the current scientific literature, the use of treadmill walking seems to be more beneficial in PD subjects, and if feasible, should be recommended over cycle training.

\section{References}

Arcolin, I., Pisano, F., Delconte, C., Godi, M., Schieppati, M., Mezzani, A., Picco, D., Grasso, M., \& Nardone, A. (2015). Intensive cycle ergometer training improves gait speed and endurance in patients with Parkinson's disease: A comparison with treadmill training. Restorative Neurology and Neuroscience, 34, 125-138.

Bello, O., Sanchez, J.A., Lopez-Alonso, V., Márquez, G., Morenilla, L., Castro, X., Giraldez, M., Santos-García, D., \& Fernandez-del-Olmo, M. (2013). The effects of treadmill or overground walking training program on gait in Parkinson's disease. Gait Posture, 38, 590-595

Lauhoff, P., Murphy, N., Doherty, C., \& Horgan, N.F. (2013). A controlled clinical trial investigating the effects of cycle ergometry training on exercise tolerance, balance and quality of life in patients with Parkinson's disease. Disabil Rehabil. $35,382-387$.

Morris, M.E., Iansek, R., Matyas, T.A., \& Summers, J.J. (1996). Stride length regulation in Parkinson' s disease Normalization strategies and underlying mechanisms. Brain, 119, 551-568.

Shulman, L.M., Katzel, L.I., Ivey, F.M., Sorkin, J.D., Favors, K., Anderson, K.E., Smith, B.A., Reich, S.G., Weiner, W.J., \& Macko, R.F. (2013). Randomized clinical trial of 3 types of physical exercise for patients with Parkinson disease. JAMA Neurology, 70, 183-190.

Smania, N., Corato, E., Tinazzi, M., Stanzani, C., Fiaschi, A., Girardi, P., \& Gandolfi, M. (2010). Effect of balance training on postural instability in patients with idiopathic Parkinson's disease. Neurorehabil Neural Repair, 24, 826-834.

Wong-Yu, I.S., \& Mak, M.K. (2915). Multi-dimensional balance training programme improves balance and gait performance in people with Parkinson's disease: A pragmatic randomized controlled trial with 12-month follow-up. Parkinsonism Relat Disord, 21, 615-621.

Yang, W.C., Wang, H.K., Wu, R.M., Lo, C.S., \& Lin, K.H. (2015) Home-based virtual reality balance training and conventional balance training in Parkinson's disease: A randomized controlled trial. J Formos Med Assoc, S0929-6646(15)00249-1. 